\title{
Electrochemical Determination of Tyrosine in Human Serum Based on Glycine Polymer and Multi-walled Carbon Nanotubes Modified Carbon Paste Electrode
}

Zhiqiang Wei, Yongjian Sun, Qianwen Yin, Liuxue Wang, Shuaigao Chen, Rui Sheng, Dengyun Pan, Hui Yang ${ }^{*}$, Sanqiang Li ${ }^{*}$

Collage of Medical, Henan University of Science and Technology, Luoyang, 471023, China.

*E-mail: yanghui7761@163.com, sanqiangli2001@163.com

doi: $10.20964 / 2018.08 .26$

Received: 23 April 2018 / Accepted: 25 May 2018 / Published: 5 July 2018

A modified carbon paste electrode (CPE) for the sensitive detection of tyrosine (Tyr) in human serum has been fabricated by glycine polymer (Poly(Gly)) and multi-walled carbon nanotubes (MWCNTs). Poly(Gly) was prepared by in situ electrochemical polymeric deposition. The operational parameters of electrochemical polymeric deposition such as concentration of Gly solution and CV scan cycles which affected the analytical performance of the modified electrode were optimized. Compared to the bare CPE and MWCNTs modified CPE, Poly(Gly)/MWCNTs/CPE exhibited excellent electrooxidation towards Tyr. Under the optimized conditions, the value of the linear sweep voltammetry (LSV) oxidation peak current was linearly related with $0.2 \sim 400 \mu \mathrm{M}$ Tyr, with the detection limit of $0.07 \mu \mathrm{M}$ (S/N=3). Furthermore, Poly(Gly)/MWCNTs/CPE was applied to the determination of Tyr in diluted serum samples.

Keywords: Tyrosine; Glycine polymer; Multi-walled carbon nanotubes; Electrochemical polymeric deposition; Carbon paste electrode.

\section{$\underline{\text { FULL TEXT }}$}

(C) 2018 The Authors. Published by ESG (www.electrochemsci.org). This article is an open access article distributed under the terms and conditions of the Creative Commons Attribution license (http://creativecommons.org/licenses/by/4.0/). 